\title{
DDGS affordances for value creation
}

\author{
Claudio Vitari and Federico Pigni \\ Grenoble Ecole de Management, Grenoble, France \\ \{claudio.vitari, federico.pigni\}@grenoble-em.com
}

\begin{abstract}
The world is changing, and is changing thanks to the data. New opportunities for business action and service creation are emerging from the massive availability of real-time digital data. We characterize these opportunities proposing a framework rooted in the Technology Affordance theory studying the dynamic interactions between people and organizations, and the technological artifact. This approach provided encouraging results in understanding the dynamic nature of IT based innovation.
\end{abstract}

Keywords: Digital Data Genesis and Streaming, affordances, intents, technological capabilities, value creation, service engineering, service management.

\section{$1 \quad$ Research introduction}

The constant interaction of users with devices embedding digital capabilities both voluntary and unconsciously is a reality that has far reaching implications for individuals and human relationships, always and everywhere connected [1]. Volumes of data are born in digital form both as a result of humans' and devices' activities (Digital Data Genesis). Current studies in the Information Systems domain approach the phenomenon from a data management perspective, mainly focusing on the concept of big data. The core reasoning of these studies is that organizations now have the opportunity to collect, process, and store ever-growing and complex datasets to support their decision making processes and strategies. These large and complex databases require specific storage, management, analysis, and visualization technologies to be effectively used, such as: business intelligence and analytics tools to dive and make sense of big data [2,3]. Very few studies tried to explain the strategic opportunities for extracting value from the real-time streams of digital data that are being created in massive quantities other than through analytics [4]. We think that

adfa, p. 1, 2011

(C) Springer-Verlag Berlin Heidelberg 2011 
new forms of value creation emerge from the interplay of real time data streams and a firm's complementary resources [4]. To understand the uses and consequences of real time data streams we adopt a framework rooted in Technology Affordances theory and we consider the dynamic interactions between people and organizations - the social actors -, and the technological artifact. In this paper we will present new conceptual tools to understand and profit from real time digital data streams.

\subsection{The research perspective: affordances}

Recent research has identified the interplay of social actors' intents and technology capabilities as at the origin of customer value and the consequent success of technological innovations and services $[5,6]$. It is from this interplay that uses and customer value emerge - whether planned or serendipitous. Uses and value could be conceived and expected by design or emerge unexpected with use, in either case what emerges are unique capabilities of the bundle that neither the technology nor the social actor alone could exercise [7]. These uses are named Affordances [8]. An Affordance represents the "opportunities for action" as perceived by a social actor in its environment. Moreover, social actors perceive differently the possibilities of an artifact to afford an action. As a possibility for action, rather than the action itself, an Affordance is conceptually separate from a given behavior, and it is the necessary precondition to it. The affordance perspective assumes that a technological artifact has some recognizable functionalities but that their analysis "in-use" has to account for the awareness of social actors' intentions, thus recognizing the object's social nature [8-10]. Thanks to this concept, the materiality of an object and a social actor's perceptions are viewed relationally and jointly in terms of how each favors or shapes actions.

In Information Systems, affordances are the result of the confluence or intertwining of the capabilities provided by 
information and communication technologies and the actions taken by the social actors using them [11]. In this context, the embedding of digital capabilities in artifacts is indeed seen as a manifestation of the digital materiality of the object itself [1].

We adopted this alternative research perspective because we aim to take a fresh look at the interplay between technology and people, but to do so as in an emerging phenomenon. Affordances allow us to overcome some of limitations of current management theories [15]:

1. that ignore the possibility that humans using technology can enact new practices or outcomes by focusing only on psychological or social behavior;

2. that technology can produce and have unintended uses, thus overcome simplistic or deterministic assumptions about the effects of technology on human and organizational outcomes.

The affordance concept is then promising for gaining a deeper understanding of the possible uses of information technology to engineer and manage services. In so far as we are interested in engineering and managing services we take into consideration affordances that make innovative digital services possible, referring to them as Service Affordances. We consider that the current technological capability to digitally represent and stream actions in real-time (DDG and DDS) provides the opportunity for new service value creation.

\section{$2 \quad$ Research focus}

In this section we focus our attention on the specific category of Service Affordances emerging from Digital Data Genesis and Stream (DDGS). Our main interest is related to the opportunities for servicing customers emerging from organizational intents, technological capabilities, and individuals. 


\subsection{Defining Digital Data Genesis and Stream.}

We define Digital Data Genesis as the real-time inception of an informational representation of an event or state of an entity in digital form. The defining characteristic of this phenomenon is that data are born digital: the timing of digital data generation is concurrent with the event that the digital data represent $[12,13]$. The fact of a restaurant waiter taking a food order with a palm electronic device as soon as a customer expresses his wishes is a simple example of DDG. The digital data is the information representation of the food order and its creation is concurrent with the customer's order formulation. It's also DDG when the customer her-self passes the order using a touch screen installed at the table. DDG is a different concept from digitalization. Back to the example, DDG would not occur if the order is taken on paper and subsequently input into the electronic cash register. In this case the data existed in another form before becoming digital, data was not born digital but digitalized.

As a consequence, DDG enables information representations of real objects and events, without significant delays (in near realtime). In addition, the digital form of these information representations facilitates their interaction, manipulation and communication in the electronic information space. As previously defined, DDG activity is separated from any consecutive information processing or more sophisticated analytics. Digital Data Streams (DDS) are composed by the flow of data originating from DDG activities and can be intercepted and used by organizations or individuals [4]. Rather than focusing on technical specifications (push versus pull, RSS versus messaging), we characterize a DDS pragmatically as a data source that is constantly (or very frequently) changing and evolving. A database that is accessible in real time through an API and is updated as new data becomes available is an instantiation of a digital data stream. Therefore, the defining characteristics of a DDS are timeliness and a continuous flow of DDG events. The prototypical 
instance of a DDS is one in which DDG events are streamed continuously in real-time. Twitter is an example: each tweet is a DDG event and the tweet-stream, eventually accessible through API, is a DDS. In conclusion, the combination of DDG and DDS makes Digital Data Genesis and Stream (DDGS): the flow of realtime inceptions of informational representations of events or states of entities in digital form that can be intercepted and used by organizations or individuals. For example, a single click on a hyperlink is the digital representation of a person's decision (a DDG event). Activity on a website is a stream of personal decisions, and is aptly called the clickstream.

\section{Service Affordances from DDGS}

The application of the affordance perspective on DDGS phenomena facilitates the comprehension of the interplay of a social actor's intents with the flow of real-time digital data and the respective technological capabilities that carry it out. The principal social actor's intents contributing to the emergence of service affordances are about the intent to provide value adding services. This intent, stemming from the technological capabilities of DDG and DDS, interacts with the customer's willingness to be serviced. Value is created when the social actor is able to enact the Service Affordances to increase the customer's willingness to pay for its offers or reduce the opportunity cost of the resources it needs to create existing value propositions. The principal technology capabilities contributing to the emergence of Service Affordances from DDGS concern ever more pervasive digital data acquisition, near real-time processing, and telecommunicating

systems. In practice, the interplay of these digital capabilities and the above-mentioned social actors' intents results in the Service Affordances. The combination of the multitude of these social actors' intents with the multiplicity of technologies having these capabilities determines infinite Service Affordances: from the waiter's use of palm devices to register and stream food orders to 
the restaurant kitchen, to worldwide financial high frequency trading round the clock. Hence, we have decided to focus on the affordances that create value in radically new and unique ways. Through an analysis of around 60 different empirical business examples, we identified four of them: Sensing, Mass Sensing, Experimenting, and Coordinating.

\subsection{Sensing}

We define Sensing as the affordance gained by detecting in realtime the current state of a single given entity (human, object, event...). Examples are the location of a single airplane, the speed of a specific car, or the mood of an individual person. We consider this affordance to be at the base of all the new value creation opportunities offered by DDGS, making it a first-order affordance, as the other DDGS affordances are built on Sensing. Effectively, Web 2.0 and social networks have allowed the creation of new ways for people to express themselves in real time. 4Square, for example, provides Sensing on other people location and "exploration" behavior. Users are uniquely identified and traced through DDG capabilities ("the check-in" and the GPS data). Sensing is an affordance emerging also from machine-tomachine (M2M) data streams as electronic devices have an unprecedented ability to automatically and continuously sense the environment, and automatically react to the incoming flow of data. Examples are the automatic alerting system at an air traffic control center in case of specific flight path deviation or the automatic cruise control of the speed of a car. The technological capabilities that support Sensing affordance include the diffusion of electronic identifiers (RFID chips, flash-tag...) and relative readers (smart phones, RFID readers, cameras...) or those technologies providing the possibility to communicate a status update, and the processing capability to analyze the characteristics of the single entity in near real-time. These characteristics can be brought along by the entity it-self or remotely stored elsewhere 
and accessed at the moment of the individual identification. The sensing intent is related to the social actors' intention to exceed current bounded perception related to their own sphere of experience. By tapping into a wider characterization of digitally represented entities cognition is extended beyond traditional physical barriers. Well-established examples relate to the Wal*Mart mandate to its suppliers to apply RFID tags to every single product or to all cattle in Canada and Europe.

\subsection{Mass Sensing}

We define Mass Sensing as the affordance gained by identifying the state of multiple entities in real time, altogether, contextualized by their relationships. Mass Sensing is a secondorder affordance based on the Sensing affordance. For example, if real-time sensing makes it possible to locate a single vehicle, it is therefore possible to sense all the cars on a road and traffic congestion could be detected. Both TomTom (http://www.tomtom.com) and Inrix (http://www.inrix.com) are examples of how sensing a mass of vehicles can be used to provide added-value services to drivers in the form of real-time traffic information and more efficient routes. Both companies aggregate information from a multitude of DDS sources, including telecom operators, road sensors, and navigation systems readings. The technological capability that supports Mass Sensing is related to the development of rule-based and filtering functionalities and of complex event processing engines. The joint effect of the capability to filter relevant information and process them provides the opportunity to "sense" and evaluate multiple and meaningful concurrent events. Through these technological capabilities, each entity of the population is uniquely identifiable and distinguishable. The availability online of massive information concerning products, services or user behaviors effectively determines the potential for comparing and choosing based on data available for the entire population. The Sensing 
intent is related to the social actors' intention to track and trace entire entity populations both in time and space. In business this intent is pursued with the aim of optimizing business processes and streamline activities, and of exerting market power. This intent typically resulted in specific strategic initiatives deployed to increase the control of product and services along supply chains or in retail surfaces or for monitoring resources and capacities (empty seats for airliners, number of passengers, flow of people, products on the shelves, etc.).

\subsection{Experimenting}

We define Experimenting as the affordance gained by fast cycling data generation and streaming on the entity with actions on this measured entity or its environment. Experimenting is a secondorder affordance based on the Sensing affordance. A/B tests on web pages for selecting a layout, or the massive experimentation ongoing in major websites, are examples of this affordance. The Experimenting affordance makes it possible to test and have immediate feedback on business decisions, from the change of a webpage layout to more complex information. New Brands Analytics provides a service that extracts specific feedback from customers' unstructured mentions on social media channels. Firms can then adjust their behaviors in real time, correct any shortcomings, and monitor the outcomes. At the same time, they can experiment with different configurations of the service and fine-tune it on the basis of customers' mentions. The technological capabilities that support this affordance include, in addition to the capabilities required for Sensing affordance (electronic identifiers, readers, processing capability), the actuators (software as well as hardware: thermostats, motors, electro-active polymers...) located in the sensed environment. The social actors' intents that support this affordance are related to the wish to understand the material reality. This wish to understand takes the specific form of the empirical method that 
arbitrates between competing theories, models and hypotheses through experimental science. The combination of these technological capabilities and human intents allows the emergence of Experimenting.

\subsection{Coordinating}

We define coordinating as the affordance gained by adjusting a behavior based on fast cycle feedback regarding the current state of other entities. Coordinating is a second-order affordance based on the Sensing one. This affordance is at the base of services such as Foursquare Radar, which enables users to coordinate spontaneously with friends by "sensing" their presence in the area. The technological capabilities that support this affordance include the diffusion of electronic communication devices (mobile phones, computers...) and the telecommunication infrastructure (Internet network, GSM network...) making the information exchange between these devices possible. Moreover, the availability of virtual networks - both social and machine to machine networks - provides the logical infrastructure for the exchange and the digital coordination to emerge. The social actors' intents that support this affordance are related to the willingness to coordinate with the other social actors as well as artifacts. This peer-to-peer approach spreads out together with the principle of human equality and mutual aid in order to achieve objectives beyond the capability of the single individual. The combination of these technological capabilities and human intents allows the emergence of the Coordinating affordance. An example of this affordance comes from the Arab countries where protesters used electronic communication devices (and applications like Twitter and Facebook) to coordinate and rally their supporters without a real or organized coordination [14]. 


\section{Discussion and conclusions}

The affordance perspective facilitates the understanding of the interplay of people or organizational intents and technology capabilities in customer value creation and the consequent success of technological innovations and services. The value of an affordance approach lies clearly in the relational definition of the "affordance" concept linking actors and technology. In a society where an increasing number of activities of public and private organizations are engineered and managed as services, the affordance perspective can be particularly valuable for innovation and competitiveness making managers better "system thinkers" [15]. IT and Information systems are at the core of this transformation. Specifically, the crucial role played by data through the development of pervasive computing, the spreading of sensors and the increase in the creation, storage, communication and processing of information - incites researchers to study DDGS. In particular, we identify central research questions that could be explored to enhance understanding of the levers for improving service development, engineering and management based on DDGS, through the affordance perspective: What are the levers and barriers in the emergence of DDGS affordances? How do individuals and organizations recognize DDGS affordances? How do individuals and organizations select DDGS affordances for services? To what extent do the organization's capabilities influence service development, engineering and management based on DDGS affordances? We will start answering some of these questions by deepening our knowledge on the around 60 business cases we identified for our four service affordances.

\section{References}

1. Yoo, Y., Bol, R.J., Jr, Lyytinen, K., Majchrzak, A.: Organizing for Innovation in the Digitized World. Organ. Sci. 23, 1398-1408 (2012). 
2. Chen, H., Chiang, R., Storey, V.: Business Intelligence and Analytics: From Big Data to Big Impact. Manag. Inf. Syst. Q. 36, 1165-1188 (2012).

3. Pospiech, M., Felden, C.: Big Data - A State-of-the-Art. AMCIS 2012 Proc. (2012).

4. Piccoli, G., Pigni, F.: Harvesting External Data: The Potential of Digital Data Streams, $\mathrm{http}: / / \mathrm{misqe}$.org/ojs2/index.php/misqe/article/view/475, (2013).

5. Jung, J.H., Schneider, C., Valacich, J.: Enhancing the Motivational Affordance of Information Systems: The Effects of Real-Time Performance Feedback and Goal Setting in Group Collaboration Environments. Manag. Sci. 56, 724-742 (2010).

6. Zammuto, R.F., Griffith, T.L., Majchrzak, A., Dougherty, D.J., Faraj, S.: Information Technology and the Changing Fabric of Organization. Organ. Sci. 18, 749-762 (2007).

7. Nevo, S., Wade, M.: The formation and value of IT-enabled resources: antecedents and consequences of synergistic relationships. MIS Q. 34, 163-183 (2010).

8. Hutchby, I.: Technologies, Texts and Affordances. Sociology. 35, $441-456$ (2001).

9. Fayard, A.-L., Weeks, J.: Photocopiers and Water-coolers: The Affordances of Informal Interaction. Organ. Stud. 28, 605-634 (2007).

10. Montesano, L., Lopes, M., Bernardino, A., Santos-Victor, J.: Leaming Object Affordances: From Sensory-Motor Coordination to Imitation. IEEE Trans. Robot. 24, 15-26 (2008).

11. Majchrzak, A.: Fostering Innovation and Intellectual Capital Creation: The Paradoxical Influence of Social Media Affordances. (2011).

12. Vitari, C., Piccoli, G., Mola, L., Rossignoli, C.: Antecedents of IT Dynamic Capabilities in The Context Of The Digital Data Genesis. ECIS 2012 Proc. (2012).

13. Raguseo, E., Vitari, C., Piccoli, G.: Gaining Competitive Advantage from Digital Data Genesis Dynamic Capability and the Moderating Role of Environmental Turbulence. itAIS Conference proceedings. , Rome, Italy (2012).

14. Christensen, C.: Twitter Revolutions? Addressing Social Media and Dissent. Commun. Rev. 14, 155-157 (2011).

15. Majchrzak A., Markus M. L., Technology Affordances and Constraints in Management Information Systems (MIS), in E. Kessler (Ed.), Encyclopedia of management theory. (pp. 832-836). Thousand Oaks, CA: SAGE Publications, Inc. doi: http://dx.doi.org/10.4135/9781452276090.n256 (2013). 